\title{
Potencial forrajero de líneas experimentales de soya (Glycine max)
}

\section{Forage potential of experimental soybean lines (Glycine max)}

\begin{abstract}
Jonathan Raúl Garay-Martínez 1 (1D) Nicolás Maldonado-Moreno [D] Guillermo Ascencio-Luciano ${ }^{1}$ (D) Santiago Joaquín Cancino ${ }^{2}$ (D) Yuridia Bautista-Martínez $z^{3}$ (D) Lorenzo Danilo GranadosRivera ${ }^{4}$ (D)

${ }^{1}$ Campo Experimental Las Huastecas, Instituto Nacional de Investigaciones Forestales, Agrícolas y Pecuarias. Carretera Tampico-Mante km 55, Villa Cuauhtémoc, CP. 89610. Altamira, Tamaulipas, México.

${ }^{2}$ Facultad de Ingeniería y Ciencias, Universidad Autónoma de Tamaulipas. Centro Universitario Campus Victoria, Edificio Centro de Gestión del Conocimiento $4^{\circ}$ piso. CP. 87120 .Cd. Victoria, Tamaulipas, México.

${ }^{3}$ Facultad de Medicina Veterinaria y Zootecnia "Dr. Norberto Treviño Zapata", km 5. Carretera VictoriaMante, Ciudad Victoria, CP. 87000 Tamaulipas, México.

${ }^{4}$ Campo Experimental General Terán, Instituto Nacional de Investigaciones Forestales, Agrícolas y Pecuarias. Carretera Montemorelos-China km 31, Exhacienda Las Anacuas, CP. 67400. General Terán, Nuevo León, México.

*Autor de correspondencia: granados.danilo@inifap.gob.mx

Artículo científico Recibido: 01 de marzo 2021 Aceptado: 01 de octubre 2021

RESUMEN. Se evaluaron cinco líneas experimentales (H15-0625, H15-0249, H14-0307, H14-0496 y H10-0556) y una variedad comercial (Huasteca 300) de soya [Glycine max (L.) Merr.] a 77, 91 y 105 días después de la siembra (dds). Las variables fueron tasa de crecimiento del cultivo (TC), rendimiento de materia seca total (RMST) y contenidos de proteína cruda (PC), nutrientes digestibles totales (NDT) y energía metabolizable (EM). Se utilizó un diseño completamente al azar con cuatro repeticiones (Tukey; $\alpha=0.05$ ). Las líneas H10-0556 y H14-0496, presentaron las mayores TC $(p<0.05)$, las cuales en promedio fueron de 100 , 132 y $123 \mathrm{~kg} \mathrm{ha}^{-1}$ día $^{-1}$; mientras que H10-0556 y H14-0496 presentaron mayor RMST, 7.689, 11.981 y $12.921 \mathrm{t} \mathrm{ha}^{-1}$, a los 77, 91 y 105 dds, respectivamente. Los materiales H14-0496, H15-0625 y Huasteca 300 mostraron mayor NDT ( $p<$ 0.05), en promedio 580, 654 y $708 \mathrm{~g} \mathrm{~kg}^{-1}$ a los 77, 91 y $105 \mathrm{dds}$, respectivamente. La mayor EM la presentaron H14-0496, H15-0625 y Huasteca 300, 2.57 Mcal $\mathrm{kg}^{-1}$, a los 105 dds. Las líneas experimentales H10-0556, H14-0307 y H14-0496, presentaron el mejor potencial forrajero en comparación con la variedad comercial debido al mayor rendimiento de forraje y valor nutritivo; estas líneas podrían cosecharse entre 91 y 105 dds, manteniendo el valor nutritivo del forraje.

Palabras clave: Energía, leguminosa, rendimiento de forraje, soya forrajera, valor nutritivo.

ABSTRACT. Five experimental lines (H15-0625, H15-0249, H14-0307, H140496, and H10-0556) and a commercial variety (Huasteca 300) of soybean [Glycine $\max$ (L.) Merr.] were evaluated at 77, 91, and 105 days after sowing (das). The variables were crop growth rate (GR), total dry matter yield (TDMY), and crude protein (CP), total digestible nutrients (TDN) and metabolizable energy (ME). A completely randomized design with four replicates (Tukey; $\alpha=0.05$ ) was used. Lines $\mathrm{H} 10-0556$, and H14-0496 presented the highest GR $(\mathrm{p}<0.05)$, which on average were 100,132 , and $123 \mathrm{~kg} \mathrm{ha}^{-1}$ day $^{-1}$; while $\mathrm{H} 10-0556$, and $\mathrm{H} 14-0496$ showed higher TDMY, 7.689, 11.981, and $12.921 \mathrm{t} \mathrm{ha}^{-1}$, at 77, 91, and 105 das, respectively. The materials $\mathrm{H} 14-0496, \mathrm{H} 15-0625$, and Huasteca 300 showed higher TDN ( $\mathrm{p}<0.05$ ), on average 580,654, and $708 \mathrm{~g} \mathrm{~kg}^{-1}$ at 77, 91, and 105 das, respectively. The highest ME was presented by $\mathrm{H} 14-0496, \mathrm{H} 15-0625$, and Huasteca 300, $2.57 \mathrm{Mcal} \mathrm{kg}^{-1}$, at 105 das. Experimental lines $\mathrm{H} 10-0556, \mathrm{H} 14-0307$, and $\mathrm{H} 14-0496$, presented the best forage potential in comparison with the commercial variety due to the higher forage yield and nutritional value; these lines could be harvested between 91 and 105 das, maintaining the nutritional value of the forage.

Key words: Energy, forage yield, forage soybean, legume, nutritional value.
\end{abstract}

Como citar: Garay-Martínez JR, Maldonado-Moreno N, AscencioLuciano G, Cancino SJ, BautistaMartínez Y, Granados-Rivera LD (2021) Potencial forrajero de líneas experimentales de soya (Glycine max). Ecosistemas y Recursos Agropecuarios Núm. Esp. II: e2932. DOI: 10.19136/era.a8nll.2932 


\section{INTRODUCCIÓN}

La ganadería es una actividad importante que genera empleo y contribuye a la seguridad alimentaria de la población (Arieta 2020). En los sistemas de producción pecuarios, la base de la alimentación de los rumiantes es el forraje; este es un alimento de bajo costo, en particular, para sistemas de producción extensivos (Bautista-Martínez et al. 2020).

Por otro lado, la producción ganadera tiene dificultades, entre las que destaca la falta de alimento, aunado a una deficiente nutrición; lo anterior, se debe en forma principal a que los pastos de las zonas de escasa precipitación en México, como Dichanthium aristatum (Ávila 2013) y Pennisetum ciliare (GarayMartínez et al. 2018) tienen bajos rendimientos de materia seca (MS; 2 a $5 \mathrm{t} \mathrm{ha}^{-1}$ ). En estas condiciones, la producción de materia seca mayor se tiene en los meses de junio a noviembre (Ávila 2013). Al respecto, durante la época seca disminuye la producción de forraje hasta en 90\% (Garay-Martínez et al. 2018); así como el valor nutrimental de los pastos: digestibilidad $400 \mathrm{~g} \mathrm{~kg}^{-1}$ y contenidos de proteína cruda 30 a $50 \mathrm{~g} \mathrm{~kg}^{-1}$ (Ávila 2013). Lo anterior, afecta de forma negativa los parámetros productivos de las unidades de producción, ya que los rumiantes alimentados con forraje de bajo valor nutrimental $(<$ $7 \%$ proteína cruda) no aprovechan de forma eficiente la energía de la celulosa y hemicelulosa, debido a que la población de microorganismos no tiene una adecuada actividad y en consecuencia disminuye la digestión de la fibra del forraje (Belachew et al. 2013). Mientras que la inclusión de leguminosas en la dieta de estos animales incrementa su consumo de proteína mejorando de esta manera la productividad del ganado (Enríquez et al. 2011).

La soya [Glycine max (L.) Merr.] es una leguminosa que puede ser una alternativa forrajera cultivada en zonas tropicales (Ávila et al. 2014). Tiene un rendimiento de forraje (MS) entre 4.2 y $7.0 \mathrm{t} \mathrm{ha}^{-1} \mathrm{a}$ los 100 días después de la siembra (Devi et al. 2011, Ávila et al. 2014). Así mismo, presenta valores de digestibilidad de 600 a $650 \mathrm{~g} \mathrm{~kg}^{-1}$, contenidos de proteína cruda (PC) entre 120 y $200 \mathrm{~g} \mathrm{~kg}^{-1}$, fibra detergente neutro (FDN) de 449 a $457 \mathrm{~g} \mathrm{~kg}^{-1}$ y fibra deter- gente ácido (FDA) de 312 a $341 \mathrm{~g} \mathrm{~kg}^{-1}$ (Touno et al. 2014, Nkosi et al. 2016). El valor nutritivo del forraje de soya es similar al de heno de alfalfa en PC (172 a $194 \mathrm{~g} \mathrm{~kg}^{-1}$ ), FDN (446 a $531 \mathrm{~g} \mathrm{~kg}^{-1}$ ) y FDA (294 a $402 \mathrm{~g} \mathrm{~kg}^{-1}$ ) (Karayilanli y Ayhan 2016). Por ello, al forraje de soya se sitúa como un excelente complemento para animales en pastoreo, en particular, durante la época seca; aseveración que está respaldada por los resultados por estudios realizados por Ávila et al. (2014) quienes reportaron un mayor incremento de la ganancia de peso de vacas complementadas $\left(0.266 \mathrm{~kg}_{\text {animal }}{ }^{-1}\right.$ día $\left.^{-1}\right)$ en comparación con la ganancia de vacas del tratamiento sin complementación $\left(-0.111 \mathrm{~kg}\right.$ animal $^{-1}$ día $\left.^{-1}\right)$. En este mismo sentido, se ha reportado que la inclusión de $30 \%$ de la dieta integral, de forraje de soya cosechado a los 68 dds incrementó la ganancia diaria de peso en corderos, además, se optimizó la conversión alimenticia (Torres-Salado et al. 2020).

En el estado de Tamaulipas existen variedades comerciales de soya como Huasteca 200, Huasteca 300 y Huasteca 400 , que tienen el potencial para producir forraje (Ávila et al. 2014); no obstante, se debe considerar que la selección de dichas variedades fue para la producción de grano, dejando a un lado las características forrajeras. Por lo que es necesario llevar a cabo la evaluación y selección de materiales que tengan alternativas forrajeras para la ganadería tropical del país. Con base en lo anterior, el objetivo del presente estudio fue evaluar el rendimiento de biomasa, morfología y composición química de cinco líneas experimentales de soya a diferentes días de cosecha en condiciones de riego.

\section{MATERIALES Y MÉTODOS}

\section{Localización del estudio y características edafo- climáticas}

El estudio se realizó de agosto a noviembre de 2019 en el Sitio Experimental Ébano. Las coordenadas geográficas son $22^{\circ} 10^{\prime} 05^{\prime \prime} \mathrm{N}$ y $98^{\circ} 28^{\prime} 10^{\prime \prime}$ $\mathrm{O}$, a $19 \mathrm{msnm}$. El clima se clasifica como cálido subhúmedo $\mathrm{Ax}_{2}$ ( $\mathrm{x}^{\prime}$ ) (García 2004). Las temperaturas máxima y mínima promedio son de 30.4 y $20.1{ }^{\circ} \mathrm{C}$, respetivamente; la precipitación promedio anual del 
lugar es de $836 \mathrm{~mm}$ y el $78 \%$ se presenta en los meses de junio a octubre (Figura 1).

\section{Tratamientos}

Se utilizaron cinco líneas experimentales y como testigo (Tabla 1) una variedad comercial de soya [Glycine max (L.) Merr.], las cuales se evaluaron cada 14 días, desde los 77 hasta los 105 días después de la siembra (dds).

\section{Establecimiento y manejo del cultivo}

La preparación de suelo y control de malezas y plagas se realizó de acuerdo con el paquete tecnológico para la producción de soya para el sur de Tamaulipas (Maldonado 2017). La siembra se realizó el 19 de agosto del 2019 bajo condiciones de riego, en surcos a $0.76 \mathrm{~m}$ de distancia. En un metro lineal hubo $20 \pm 2$ plantas y con ello se obtuvo una densidad de aproximada de 250000 plantas ha ${ }^{-1}$. Se utilizaron 28 parcelas, compuestas de cuatro surcos de $5 \mathrm{~m}$ de largo; en las cuales se realizaron tres muestreos destructivos (77, 91 y 105 dds). En cada muestreo se cosechó el forraje presente en $1 \mathrm{~m}$ lineal (unidad experimental) de cada surco a una altura 5 $\mathrm{cm}$ sobre el nivel del suelo. Posteriormente se pesó para determinar el rendimiento de forraje verde y después se tomó una submuestra de aproximadamente de $300 \mathrm{~g}$, que se separó en los componentes morfológicos: hoja, tallo y vaina (valvas + semillas) y finalmente todas las muestras se colocaron en una estufa de aire forzado a $65{ }^{\circ} \mathrm{C}$ por $72 \mathrm{~h}$. Los componentes morfológicos se pesaron antes y después de secarse para determinar el contenido de MS.

\section{Variables evaluadas}

Con el forraje cosechado en cada unidad experimental se estimó el rendimiento de materia seca total (RMST; $\mathrm{t} \mathrm{ha}^{-1}$ ) y posteriormente se calculó la tasa de crecimiento (TC; $\mathrm{kg} \mathrm{ha}^{-1} \mathrm{~d}^{-1}$ ) con la fórmula: $\mathrm{TC}=\frac{R M S T}{t}$. Donde: $\mathrm{RMST}=$ rendimiento de materia seca total $\left(\mathrm{kg} \mathrm{ha}^{-1}\right)$ y $\mathrm{t}=$ tiempo transcurrido desde la siembra a cada fecha de corte (d). También se estimó el rendimiento ( $t \mathrm{ha}^{-1}$ ) de los componentes morfológicos: hoja, tallo y vaina. Los contenidos (g $\mathrm{kg}^{-1}$ ) de proteína cruda (PC), carbohidratos no fi- brosos (CNF), grasa cruda (GC) y cenizas (Cen) se determinaron mediante la metodología descrita por la AOAC (2019). Los nutrientes digestibles totales (NDT) y la energía (Mcal $\mathrm{kg}^{-1}$ ) neta de lactancia (ENI), mantenimiento $(E N m)$, ganancia (Eng) y metabolizable (EM) se estimaron usando el modelo de la AFRC [Agricultural and Food Research Council (AFRC 1993)]. Los contenidos $\left(\mathrm{g} \mathrm{kg}^{-1}\right)$ de fibra detergente neutro (FDN) y fibra detergente ácido (FDA), se determinaron mediante el procedimiento descrito por Van Soest et al. (1991).

\section{Análisis estadístico}

Las variables se analizaron con el procedimiento GLM de SAS bajo un diseño completamente al azar con cuatro repeticiones con arreglo de parcelas divididas, donde la parcela grande fue el muestreo (77, 91 y 105 dds) y la parcela chica los materiales de soya. Cuando se encontró diferencia estadística, se aplicó la prueba de comparación de medias de Tukey $(\alpha=0.05)$.

\section{RESULTADOS}

\section{Tasa de crecimiento del cultivo}

Se observaron diferencias en la TC entre los materiales evaluados en cada fecha de corte $(p<$ 0.05; Figura 2). Las líneas experimentales $\mathrm{H} 10-0556$ y H14-0496 presentaron las mayores TC $(p<0.05)$, que en promedio fueron de 100, 132 y $123 \mathrm{~kg} \mathrm{ha}^{-1}$ día $^{-1}$ y superaron a la variedad Huasteca 300 en 57 , 56 y $51 \%$, a los 77, 91 y 105 dds, respectivamente (Figura 2). Asimismo, la TC incrementó $34 \%$ de 77 a 91 dds $(p<0.05)$ y disminuyó $5 \%$ de 91 a 105 dds ( $p$ $<0.05$, Figura 2).

\section{Rendimiento de materia seca total y de compo- nentes morfológicos}

Se registró mayor RMST a mayor edad de la planta, donde H14-0496 (13.13 $\mathrm{t} \mathrm{ha}^{-1}$ ) y H10-0556 $\left(12.71 \mathrm{t} \mathrm{ha}^{-1}\right)$ mostraron mayor biomasa $(\mathrm{p}<0.05)$. Por otro lado, se observó mayor rendimiento promedio de hoja y tallo a $91 \mathrm{dds}$, mientras que el mayor rendimiento de vaina ocurrió a 105 dds $(p<0.05)$. La edad de corte tuvo un efecto significativo $(p<0.05)$ 


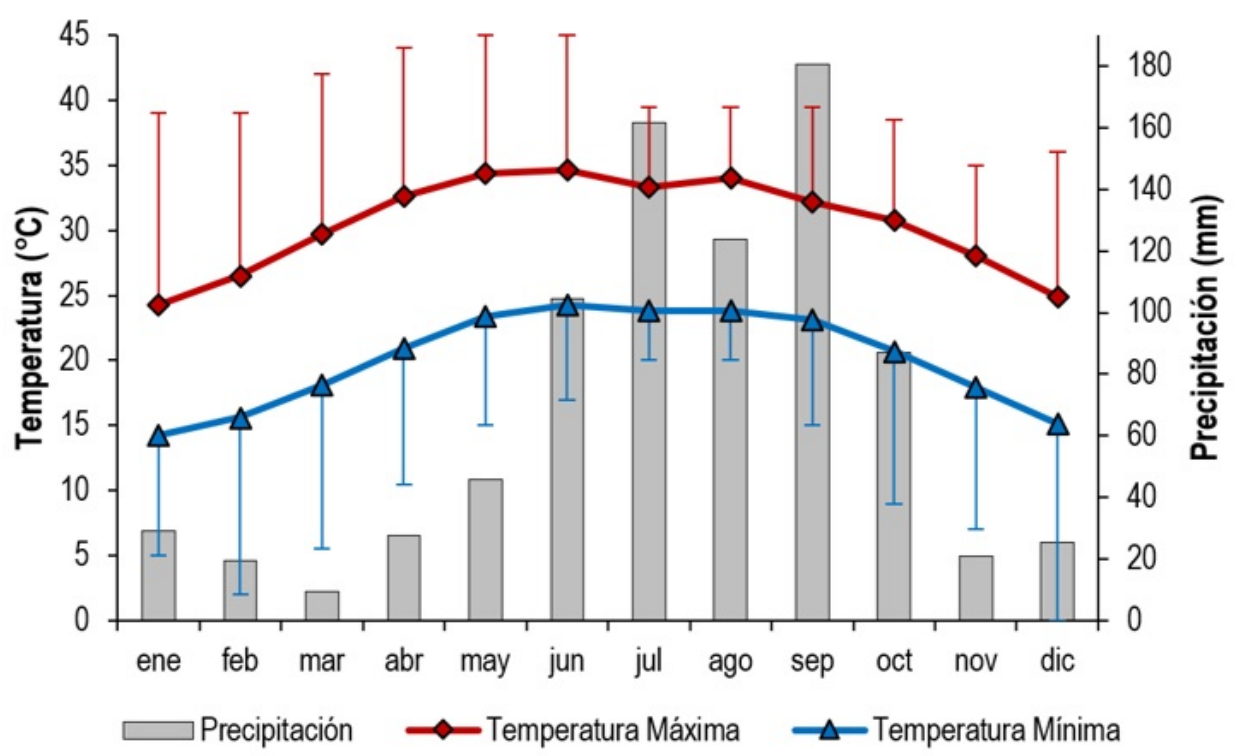

Figura 1. Distribución de la precipitación mensual acumulada, temperatura máxima y mínima mensual promedio en Ébano, San Luís Potosí. Información promedio de 29 años (1981-2010); Estación 24015 Ébano (SMN, 2020).

en el RMST, que aumentó 58 y $9 \%$, de 77 a 91 y de 91 a 105 dds, respectivamente (Tabla 2).

Tabla 1. Tratamientos experimentales y etapas reproductivas (Fehr et al. 1971).

\begin{tabular}{lcccc}
\hline \multirow{2}{*}{ Tipo } & Línea o variedad & \multicolumn{3}{c}{ Etapa reproductiva } \\
\cline { 3 - 5 } & & $77^{*}$ & $91^{*}$ & $105^{*}$ \\
\hline Experimental & $\mathrm{H} 15-0625$ & 4.0 & 6.0 & 7.0 \\
Experimental & $\mathrm{H} 15-0249$ & 4.0 & 6.0 & 7.0 \\
Experimental & $\mathrm{H} 14-0307$ & 4.0 & 5.0 & 6.0 \\
Experimental & $\mathrm{H} 14-0496$ & 5.0 & 5.5 & 6.0 \\
Experimental & $\mathrm{H} 10-0556$ & 4.0 & 5.5 & 6.0 \\
Comercial & Huasteca 300 & 5.0 & 6.0 & 7.0 \\
\hline${ }^{*}$ Días después de la siembra. & & &
\end{tabular}

En rendimiento de hoja fue mayor en todos los materiales a 91 dds $(p<0.05)$, esto sucedió con menor RMST, por tanto, al esperar 14 días para la cosecha (105 dds), aunque el rendimiento de hoja disminuye $0.853 \mathrm{t} \mathrm{ha}^{-1}$, el RMST se incrementa $9 \%$ $\left(0.952 \mathrm{t} \mathrm{ha}^{-1}\right)$. Así mismo, el menor rendimiento de hoja en cada fecha se observó en la variedad Huasteca 300 y fue superada en promedio por las líneas experimentales $\mathrm{H} 10-0556, \mathrm{H} 14-0307$ y H140496, en 96, 79 y 127\%, a los 77, 91 y 105 dds, respectivamente (Tabla 2 ).

El mayor rendimiento de tallo (en promedio) se observó en las líneas experimentales H10-
0556, H14-0496 y H14-0307 en comparación con la variedad Huasteca 300 en cada fecha de corte $(p<$ $0.05): 2.458$ vs. $0.980,3.141$ vs. 1.373 y 2.920 vs. $1.203 \mathrm{t} \mathrm{ha}^{-1}$, a los 77,91 y $105 \mathrm{dds}$, respectivamente (Tabla 2). En todos los materiales se observó que rendimiento del tallo incrementó 30\% (en promedio) de 77 a 91 dds y posteriormente disminuyó $8 \%$ ( $p<$ 0.05 ; Tabla 2). El rendimiento mayor de vaina $(p<$ 0.05) lo presentaron los materiales H14-0496 (1.158 t ha ${ }^{-1} ; 77$ dds), H15-0249 (3.802 t ha ${ }^{-1} ; 91$ dds) y Huasteca 300 (5.803 t ha $\left.{ }^{-1}, 105 \mathrm{dds}\right)$. El rendimiento de vaina de todos los materiales en promedio aumentó $299 \%$ de 77 a 91 y $71 \%$ de 91 a 105 dds ( $p<$ 0.05, Tabla 2).

\section{Composición química}

Se observó interacción en el contenido de PC entre los materiales y las fechas de corte; ya que, en el primer muestreo los materiales más sobresalientes $(p<0.05)$ fueron H14-0496, H15-0249, H15-0625 y Huasteca 300 (entre 154 y $188 \mathrm{~g} \mathrm{~kg}^{-1}$ de $\mathrm{PC}$ ). Mientras que a los 91 dds los mayores $(p<0.05)$ valores de PC los presentaron $\mathrm{H} 14-0496, \mathrm{H} 15-0249$ y Huasteca 300 (171 y $203 \mathrm{~g} \mathrm{~kg}^{-1}$ ) y en el último muestreo los materiales con mayor contenido de PC 


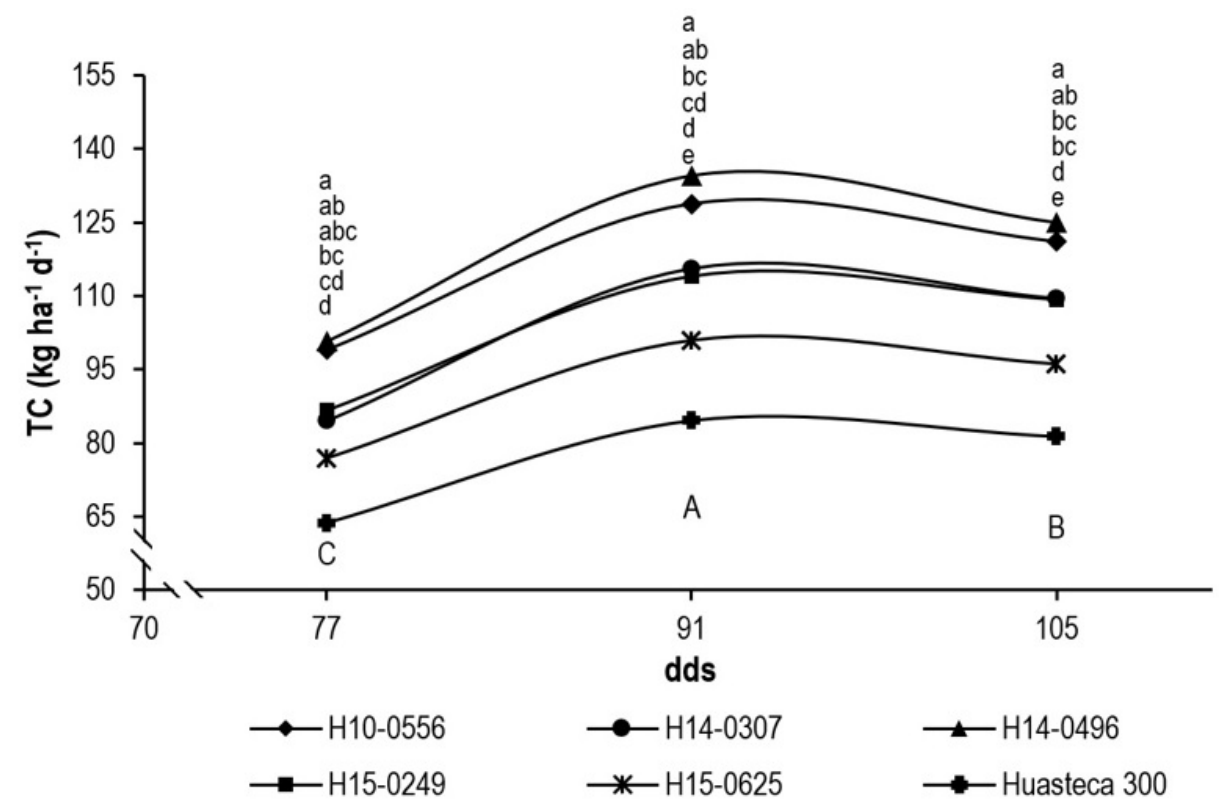

Figura 2. Tasa de crecimiento del cultivo (TC) de cinco líneas experimentales y una variedad comercial (Huasteca 300) de soya [Glycine max (L.) Merr.) a diferentes días después de la siembra (dds). Literales diferentes dentro de dds $(a, b, c, d, e)$ y entre dds $(A, B, C)$ indican diferencia estadística significativa (Tukey; $\alpha=0.05$ ).

Tabla 2. Rendimientos de materia seca total, hoja, tallo y vaina de cinco líneas experimentales y una variedad comercial (Huasteca 300) de soya [Glycine max (L.) Merr.] a diferentes días después de la siembra (dds).

\begin{tabular}{|c|c|c|c|c|c|c|c|c|c|c|}
\hline \multirow{2}{*}{ dds } & \multirow{2}{*}{ Línea/variedad } & \multirow{2}{*}{$\begin{array}{c}\text { Etapa } \\
\text { reproductiva* }\end{array}$} & \multicolumn{2}{|c|}{ RMST } & \multicolumn{2}{|c|}{ Hoja } & \multicolumn{2}{|c|}{ Tallo } & \multicolumn{2}{|c|}{ Vaina } \\
\hline & & & \multicolumn{8}{|c|}{$\mathrm{tha}^{-1}$} \\
\hline \multirow[t]{7}{*}{77} & $\mathrm{H} 10-0556$ & 4.0 & 7.620 & $a b$ & 4.633 & $a$ & 2.613 & $a$ & 0.374 & $c d$ \\
\hline & $\mathrm{H} 14-0307$ & 4.0 & 6.507 & $b c$ & 4.179 & $a b$ & 2.252 & $a b$ & 0.076 & $d$ \\
\hline & $\mathrm{H} 14-0496$ & 5.0 & 7.757 & $a$ & 4.544 & $a$ & 2.508 & $b$ & 0.705 & $b c$ \\
\hline & $\mathrm{H} 15-0249$ & 4.0 & 6.677 & $a b c$ & 3.604 & $b$ & 1.946 & $a$ & 1.126 & $b$ \\
\hline & $\mathrm{H} 15-0625$ & 4.0 & 5.913 & $c d$ & 3.359 & $b$ & 1.974 & $b$ & 0.580 & $c$ \\
\hline & Huasteca 300 & 5.0 & 4.896 & $d$ & 2.272 & $c$ & 0.980 & $c$ & 1.643 & $a$ \\
\hline & Promedio & & 6.561 & C & 3.765 & $B$ & 2.046 & C & 0.751 & C \\
\hline \multirow[t]{7}{*}{91} & $\mathrm{H} 10-0556$ & 5.5 & 11.714 & $a b$ & 6.042 & $a$ & 3.329 & $a$ & 2.343 & $b c$ \\
\hline & $\mathrm{H} 14-0307$ & 5.0 & 10.517 & $b c$ & 5.354 & $a$ & 3.044 & $a$ & 2.119 & $c$ \\
\hline & $\mathrm{H} 14-0496$ & 5.5 & 12.248 & $a$ & 4.977 & $a b c$ & 3.051 & $a$ & 4.220 & $a$ \\
\hline & $\mathrm{H} 15-0249$ & 6.0 & 10.376 & $c d$ & 4.144 & $b c d$ & 2.324 & $c$ & 3.908 & $a$ \\
\hline & $\mathrm{H} 15-0625$ & 6.0 & 9.179 & $d$ & 3.909 & $c d$ & 2.459 & $b c$ & 2.811 & $b c$ \\
\hline & Huasteca 300 & 6.0 & 7.696 & $e$ & 3.046 & $d$ & 1.373 & $d$ & 3.277 & $a b$ \\
\hline & Promedio & & 10.288 & $B$ & 4.578 & $A$ & 2.597 & $A$ & 3.113 & $B$ \\
\hline \multirow[t]{7}{*}{105} & $\mathrm{H} 10-0556$ & 6.0 & 12.711 & $a b$ & 4.800 & $a$ & 3.005 & $a$ & 4.906 & $a b c$ \\
\hline & $\mathrm{H} 14-0307$ & 6.0 & 11.499 & $b c$ & 4.461 & $a b$ & 3.024 & $a$ & 4.014 & $c$ \\
\hline & $\mathrm{H} 14-0496$ & 6.0 & 13.130 & $a$ & 4.284 & $b$ & 2.731 & $a b$ & 6.114 & $a$ \\
\hline & $\mathrm{H} 15-0249$ & 7.0 & 11.477 & $b c$ & 3.575 & $c d$ & 1.957 & $c$ & 5.945 & $a$ \\
\hline & $\mathrm{H} 15-0625$ & 7.0 & 10.085 & $d$ & 3.243 & $d$ & 2.311 & $b c$ & 4.531 & $b c$ \\
\hline & Huasteca 300 & 7.0 & 8.538 & $e$ & 1.985 & $e$ & 1.203 & $d$ & 5.350 & $a b$ \\
\hline & Promedio & & 11.240 & $A$ & 3.725 & $B$ & 2.372 & $B$ & 5.143 & $A$ \\
\hline
\end{tabular}

RMST: Rendimiento de materia seca total. Literales diferentes dentro de dds $(a, b, c, d)$ y entre dds $(A, B$, C) indican diferencia estadística significativa (Tukey; $\alpha=0.05$ ). ${ }^{*} F e h r$ et al. (1971). 
fueron $\mathrm{H} 10-0556, \mathrm{H} 14-0307, \mathrm{H} 14-0496, \mathrm{H} 15-0625$ y Huasteca 300 (entre 161 y $203 \mathrm{~g} \mathrm{~kg}^{-1}$ ). Cabe destacar que el material H15-0249 en el primer muestreo (a 77 dds) presentó uno de los valores altos de PC (188 $\left.\mathrm{g} \mathrm{kg}^{-1}\right)$, sin embargo, al final de la evaluación (a 105 dds) se registró el menor valor de PC $(p<0.05)$, esto es $160 \mathrm{~g} \mathrm{~kg}^{-1}$ (Tabla 3). No se observaron cambios $(p>0.05)$ en el contenido de $\mathrm{PC}$ entre los diferentes muestreos, el cual en promedio fue de $168 \mathrm{~g} \mathrm{~kg}^{-1}$ (Tabla 3). Lo cual indica que para defoliar por contenido de proteína podría ser en cualquier fecha en las que se cosechó el forraje, sin embargo, el mayor RMST fue a los 105 dds.

El contenido de FDN y FDA fue diferente en cada fecha y los valores mayores se observaron a menor edad de corte ( $p<0.05$; Tabla 3). El contenido celular fue menor en las líneas experimentales, ya que en promedio mostraron mayor FDN y FDA y, por tanto, la variedad 300 fue más digestible por agregar menos carbohidratos estructurales (Tabla 3). Los CNF estuvieron en menor cantidad en promedio a los 77 dds, donde la línea experimental H15-0249 superó en $23 \%$ a H10-0556 ( $<$ 0.05). El contenido de GC y NDT fue superior a mayor edad de corte, no así las cenizas ( $<<0.05$; Tabla 3 ). La cantidad mayor de energía, a los 77 dds la presentaron los materiales $\mathrm{H} 14-0307, \mathrm{H} 15-0249, \mathrm{H} 15-0625$ y Huasteca 300 ( $\mathrm{p}$ < 0.05); mientras que a los $91 \mathrm{dds}$, sobresalieron $\mathrm{H} 14-0496, \mathrm{H} 15-0249, \mathrm{H} 15-0625$ y Huasteca 300 ( $p<$ 0.05) y, por último, a los 105 dds los materiales con mayor valor energético fueron $\mathrm{H} 14-0496, \mathrm{H} 15-0625$ y Huasteca 300 (Tabla 4). De acuerdo con los resultados obtenidos (Tabla 4), los valores de ENI, ENm, ENg y EM aumentaron 15, 24, 38 y $13 \%$, respectivamente, cuando la edad de cosecha pasó de 77 a 105 dds (Tabla 4).

\section{DISCUSIÓN}

El mayor rendimiento de forraje (MS) en promedio de los siete materiales de soya a 105 dds (11.18 $\mathrm{t} \mathrm{ha}^{-1}$ ) fue superior a los reportados por Tobía y Villalobos (2004), Devi et al. (2011) y Ávila et al. (2014). En condiciones ambientales similares al lugar donde se llevó a cabo este estudio, la variedad testigo (Huasteca 300) ha tenido potencial para producir gran cantidad de forraje (Ávila et al. 2014); a pesar de lo anterior, fue superada por las líneas experimentales $\mathrm{H} 10-0556$ y H14-0496. Esto pudiera explicarse por la mayor tasa de crecimiento (Figura 2) y mayor producción de hojas de estas líneas experimentales (Tabla 2). La situación anterior, pudo generar las condiciones para que en las líneas experimentales la tasa fotosintética fuese más alta respecto a la variedad testigo (Huasteca 300); ya que se ha documentado que, a mayor biomasa de hoja existe una mayor tasa fotosintética y, en consecuencia mayor producción de forraje, como fue consignado por Pérez et al. (2004).

Por otra parte, en la selección de materiales con potencial forrajero, un aspecto importante a considerar es el rendimiento de la materia seca del tallo, ya que esta variable tiene una correlación positiva con el contenido de fibra; a mayor valor de este, la degradabilidad del forraje se reduce, por ende, la ingesta de alimento y al igual que los parámetros productivos (Vargas-Bello-Pérez et al. 2008). Al respecto, los valores de FDN y FDA en todos los materiales evaluados se encuentra dentro de un rango adecuado para ser considerados en la alimentación de rumiantes, no obstante, la línea experimental H150249 fue la que tuvo los menores valores de FDN y FDA en los periodos 77 y 91 dds, lo que indica que es un forraje poco voluminoso y con mayor contenido energético, lo cual se confirma, al considerar que fue el que tuvo el mayor contenido de EM (2.32 Mcal kg ${ }^{-1}, 77$ dds, 2.49 Mcal kg $^{-1}, 91 \mathrm{dds}$ ).

El mayor contenido de proteína de las líneas experimental evaluadas $\mathrm{H} 14-0496$ y $\mathrm{H} 15-0249$, se debe a que presentaron mayor proporción de hojas respecto a la variedad testigo (Huasteca 300) (Garay et al. 2020); en este sentido, el contenido de proteína esta correlacionado en forma positiva con la cantidad de hojas, debido a que la función fotosintética de las hojas requiere un gran contenido de $\mathrm{N}$ en comparación con otros tejidos de la planta (Shamim et al. 2021). Por ejemplo, tres cuartas partes del nitrógeno total en la hoja está relacionado con la fotosíntesis (Liu et al. 2018). Por otra parte, los contenidos promedio de PC en el periodo de evaluación de estas 
Tabla 3. Composición química de cinco líneas experimentales y una variedad comercial (Huasteca 300) de soya [Glycine max (L.) Merr.] a diferentes días después de la siembra (dds).

\begin{tabular}{|c|c|c|c|c|c|c|c|c|c|c|c|c|c|c|c|c|}
\hline \multirow{2}{*}{ dds } & \multirow{2}{*}{ Línea/variedad } & \multirow{2}{*}{$\begin{array}{c}\text { Etapa } \\
\text { reproductiva* }\end{array}$} & \multicolumn{2}{|c|}{$\mathrm{PC}$} & \multicolumn{2}{|c|}{ FDN } & \multicolumn{2}{|c|}{ FDA } & \multicolumn{2}{|c|}{ CNF } & \multicolumn{2}{|c|}{ GC } & \multicolumn{2}{|c|}{ Cen } & \multicolumn{2}{|c|}{ NDT } \\
\hline & & & & & & & & & $\mathrm{g} \mathrm{kg}$ & & & & & & & \\
\hline \multirow[t]{7}{*}{77} & $\mathrm{H} 10-0556$ & 4.0 & 153 & $b$ & 534 & $a$ & 421 & $a$ & 229 & $b$ & 18 & $b$ & 70 & $b$ & 564 & $b$ \\
\hline & $\mathrm{H} 14-0307$ & 4.0 & 150 & $b$ & 488 & $b$ & 374 & $a b c$ & 274 & $a b$ & 18 & $b$ & 73 & $a b$ & 583 & $a b$ \\
\hline & $\mathrm{H} 14-0496$ & 5.0 & 163 & $a b$ & 512 & $a b$ & 403 & $a b$ & 230 & $a b$ & 15 & $b$ & 80 & $a$ & 560 & $b$ \\
\hline & $\mathrm{H} 15-0249$ & 4.0 & 188 & $a$ & 442 & $c$ & 334 & $c$ & 283 & $a$ & 15 & $b$ & 80 & $a$ & 601 & $a$ \\
\hline & $\mathrm{H} 15-0625$ & 4.0 & 154 & $a b$ & 508 & $a b$ & 400 & $a b$ & 248 & $a b$ & 21 & $b$ & 75 & $a b$ & 578 & $a b$ \\
\hline & Huasteca 300 & 5.0 & 177 & $a b$ & 483 & $b$ & 355 & $b c$ & 237 & $a b$ & 31 & $a$ & 78 & $a$ & 601 & $a$ \\
\hline & Promedio & & 164 & $A$ & 495 & A & 381 & $A$ & 250 & $B$ & 20 & C & 76 & $A$ & 581 & $C$ \\
\hline \multirow[t]{7}{*}{91} & $\mathrm{H} 10-0556$ & 5.5 & 135 & $c$ & 445 & $a b$ & 356 & $a$ & 337 & $a$ & 23 & $d$ & 63 & $a b$ & 623 & $b c$ \\
\hline & $\mathrm{H} 14-0307$ & 5.0 & 154 & $b c$ & 463 & $a$ & 367 & $a$ & 304 & $a b$ & 18 & $d$ & 61 & $a b$ & 607 & $c$ \\
\hline & $\mathrm{H} 14-0496$ & 5.5 & 203 & $a$ & 413 & $c$ & 317 & $b$ & 263 & $b$ & 45 & $b$ & 76 & $a$ & 656 & $a b$ \\
\hline & $\mathrm{H} 15-0249$ & 6.0 & 190 & $a b$ & 416 & $b c$ & 316 & $b$ & 267 & $b$ & 58 & $a$ & 70 & $a b$ & 675 & $a$ \\
\hline & $\mathrm{H} 15-0625$ & 6.0 & 160 & $b c$ & 435 & $a b c$ & 321 & $b$ & 321 & $a b$ & 37 & $c$ & 47 & $b$ & 662 & $a b$ \\
\hline & Huasteca 300 & 6.0 & 171 & $a b c$ & 418 & $b c$ & 311 & $b$ & 306 & $a b$ & 35 & $c$ & 74 & $a$ & 643 & $a b c$ \\
\hline & Promedio & & 169 & $A$ & 431 & $B$ & 331 & $B$ & 300 & $A$ & 36 & $B$ & 65 & B & 644 & $B$ \\
\hline \multirow[t]{7}{*}{105} & $\mathrm{H} 10-0556$ & 6.0 & 170 & $a b$ & 406 & $a b$ & 330 & $a b$ & 324 & $a b$ & 39 & $c$ & 68 & $a b$ & 663 & $c$ \\
\hline & $\mathrm{H} 14-0307$ & 6.0 & 165 & $a b$ & 410 & $a$ & 352 & $a$ & 314 & $a b$ & 49 & $b c$ & 62 & $b$ & 675 & $b c$ \\
\hline & $\mathrm{H} 14-0496$ & 6.0 & 203 & $a$ & 402 & $a b$ & 291 & $b$ & 268 & $b$ & 63 & $a b$ & 65 & $a b$ & 696 & $a b c$ \\
\hline & H15-0249 & 7.0 & 160 & $b$ & 423 & $a$ & 305 & $a b$ & 298 & $a b$ & 50 & $b c$ & 68 & $a b$ & 664 & $c$ \\
\hline & $\mathrm{H} 15-0625$ & 7.0 & 161 & $a b$ & 357 & $b c$ & 288 & $b$ & 359 & $a$ & 55 & $a b c$ & 71 & $a b$ & 704 & $a b$ \\
\hline & Huasteca 300 & 7.0 & 180 & $a b$ & 347 & $c$ & 292 & $b$ & 326 & $a b$ & 72 & $a$ & 79 & $a$ & 723 & $a$ \\
\hline & Promedio & & 173 & $A$ & 391 & $C$ & 309 & C & 315 & $A$ & 55 & $A$ & 69 & $B$ & 687 & $A$ \\
\hline
\end{tabular}

PC: proteína cruda; FDN: fibra detergente neutro; FDA: fibra detergente ácido; CNF: carbohidratos no fibrosos; GC: grasa cruda; Cen cenizas; NDT: nutrientes digestibles totales. Literales diferentes dentro de dds (a, b, c, d) y entre dds (A, B, C) indican diferencia estadística significativa (Tukey; $\alpha=0.05$ ). *Fehr et al. (1971).

líneas experimentales ( 189 y $179 \mathrm{~g} \mathrm{~kg}^{-1}$ en H14-0496 y H15-0249, respectivamente) es adecuado para promover una óptima actividad microbiana en el rumen, es decir, este contenido de proteína puede generar cantidades adecuadas de péptidos, aminoácidos y amoniaco que son fuentes de nitrógeno utilizadas por los microorganismos para la síntesis de la proteína microbiana (Belachew et al. 2013).

En gramíneas forrajeras, a medida que las etapas fenológicas transcurren, se observa un incremento de biomasa vegetal, pero, con un detrimento del valor nutritivo (Enríquez et al. 2011, Garay et al. 2020). En el caso de la soya, el contenido de proteína cruda fue similar en las diferentes fechas de cosecha (Tabla 3) y a diferencia de las gramíneas, disminuyó el contenido de fibra, en consecuencia, se incrementaron los nutrientes digestibles totales (Tabla 3) y la energía (Tabla 4). Lo anterior se pudo deber, a que en el momento de cosechar el forraje (91 y 105 dds), la planta se encontraba en proceso de llenado de grano o con grano verde lleno (Tabla 1) y según Tobía et al.
(2008) y Devi et al. (2011), este componente es altamente digestible, lo cual puede explicar el alto valor nutrimental del forraje de soya. En esta investigación se observó que cuando se cosechó el forraje a los 91 y 105 dds, la mayoría de las líneas experimentales con mayor rendimiento de forraje y valor nutritivo (Tabla 2, 3 y 4) se encontraban en etapas reproductivas entre 5.0 y 6.0 (Tabla 1), que corresponden al proceso de llenado de grano o cuando el grano verde está lleno, respectivamente, según la escala de Fehr et al. (1971); lo que concuerda con otras investigaciones, en donde se menciona que en estas etapas es el momento más adecuado para cosechar el forraje de soya (Díaz et al. 2003, Tobía et al. 2008, Devi et al. 2011).

\section{CONCLUSIONES}

Con base en los resultados, las líneas experimentales evaluadas tienen el potencial para producir forraje y utilizarlo en la alimentación de rumiantes. 
Tabla 4. Energía de cinco líneas experimentales y una variedad comercial (Huasteca 300) de soya [Glycine max (L.) Merr.] a diferentes días después de la siembra (dds).

\begin{tabular}{|c|c|c|c|c|c|c|c|c|c|c|}
\hline \multirow{3}{*}{$\begin{array}{l}\text { dds } \\
77\end{array}$} & \multirow{3}{*}{$\begin{array}{l}\text { Línea/variedad } \\
\text { H10-0556 }\end{array}$} & \multirow{3}{*}{$\begin{array}{c}\begin{array}{c}\text { Etapa } \\
\text { reproductiva* }\end{array} \\
4.0\end{array}$} & \multicolumn{2}{|c|}{ ENI } & \multicolumn{2}{|c|}{$\mathrm{ENm}$} & \multicolumn{2}{|c|}{$\mathrm{ENg}$} & \multicolumn{2}{|c|}{ EM } \\
\hline & & & \multicolumn{8}{|c|}{ Mcal kg $^{-1}$} \\
\hline & & & 1.31 & $\begin{array}{ll}c \\
\end{array}$ & 1.28 & $\begin{array}{ll}c \\
\end{array}$ & 0.71 & 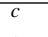 & 2.14 & $\begin{array}{ll}c \\
\end{array}$ \\
\hline & $\mathrm{H} 14-0307$ & 4.0 & 1.37 & $a b c$ & 1.36 & $a b c$ & 0.78 & $b c$ & 2.22 & $a b c$ \\
\hline & $\mathrm{H} 14-0496$ & 5.0 & 1.32 & $c$ & 1.29 & $c$ & 0.71 & $c$ & 2.14 & $c$ \\
\hline & $\mathrm{H} 15-0249$ & 4.0 & 1.44 & $a$ & 1.46 & $a$ & 0.88 & $a$ & 2.32 & $a$ \\
\hline & $\mathrm{H} 15-0625$ & 4.0 & 1.36 & $b c$ & 1.34 & $b c$ & 0.76 & $c$ & 2.20 & $b c$ \\
\hline & Huasteca 300 & 5.0 & 1.43 & $a b$ & 1.44 & $a b$ & 0.86 & $a b$ & 2.31 & $a b$ \\
\hline & Promedio & & 1.37 & C & 1.36 & $C$ & 0.78 & $C$ & 2.22 & $C$ \\
\hline \multirow[t]{7}{*}{91} & $\mathrm{H} 10-0556$ & 5.5 & 1.42 & $c$ & 1.45 & $b$ & 0.86 & $b$ & 2.28 & $b$ \\
\hline & $\mathrm{H} 14-0307$ & 5.0 & 1.42 & $c$ & 1.43 & $b$ & 0.85 & $b$ & 2.28 & $b$ \\
\hline & $\mathrm{H} 14-0496$ & 5.5 & 1.55 & $a b$ & 1.63 & $a$ & 1.03 & $a$ & 2.46 & $a$ \\
\hline & $\mathrm{H} 15-0249$ & 6.0 & 1.58 & $a$ & 1.68 & $a$ & 1.07 & $a$ & 2.49 & $a$ \\
\hline & H15-0625 & 6.0 & 1.52 & $a b$ & 1.60 & $a$ & 1.00 & $a$ & 2.42 & $a$ \\
\hline & Huasteca 300 & 6.0 & 1.49 & $b c$ & 1.56 & $a b$ & 0.96 & $a b$ & 2.39 & $a b$ \\
\hline & Promedio & & 1.49 & $B$ & 1.56 & $B$ & 0.96 & $B$ & 2.39 & $B$ \\
\hline \multirow[t]{7}{*}{105} & $\mathrm{H} 10-0556$ & 6.0 & 1.53 & $c$ & 1.62 & $c d$ & 1.01 & $c d$ & 2.44 & $c d$ \\
\hline & $\mathrm{H} 14-0307$ & 6.0 & 1.55 & $b c$ & 1.64 & $b c d$ & 1.04 & $b c d$ & 2.46 & $b c d$ \\
\hline & $\mathrm{H} 14-0496$ & 6.0 & 1.63 & $a b$ & 1.75 & $a b$ & 1.13 & $a b$ & 2.56 & $a b$ \\
\hline & $\mathrm{H} 15-0249$ & 7.0 & 1.52 & $c$ & 1.61 & $d$ & 1.00 & $d$ & 2.42 & $d$ \\
\hline & $\mathrm{H} 15-0625$ & 7.0 & 1.60 & $a b c$ & 1.73 & $a b c$ & 1.11 & $a b c$ & 2.53 & $a b c$ \\
\hline & Huasteca 300 & 7.0 & 1.66 & $a$ & 1.81 & $a$ & 1.18 & $a$ & 2.61 & $a$ \\
\hline & Promedio & & 1.58 & A & 1.69 & A & 1.08 & $A$ & 2.50 & A \\
\hline
\end{tabular}

En cuanto al mayor rendimiento de forraje y valor nutritivo, sobresalieron las líneas H10-0556, H14-0307, $\mathrm{H} 14-0496$ y $\mathrm{H} 15-0249$ y la fecha que se sugiere para cosechar estas líneas es entre 91 y 105 dds.

\section{AGRADECIMIENTOS}

Al Proyecto: 14505534871-Potencial forrajero de líneas experimentales de soya [Glycine max L. (Merr.)] para el Centro y Sur de Tamaulipas (ETAPA 1 de 2), financiado con recursos Fidecomiso-Fiscal del Instituto Nacional de Investigaciones Forestales, Agrícolas y Pecuarias.

\section{LITERATURA CITADA}

AFRC (1993) Energy and Protein Requirements for Ruminants. 1st Edition. Agricultural and Food Research Council. CAB International. United Kingdom. 176p.

AOAC (2019) Official Methods of Analysis of AOAC International. 21st Edition. Association of Official Analytical Chemists. Gaithersburg: AOAC International. USA. 700p.

Arieta RJ (2020) Ganadería: Estilo y tendencias en el nuevo sexenio. México 2000-2020. Agroproductividad 13: 29-36.

Ávila JM (2013) Producción de semilla de los pastos Angleton y Medio Bluestem. 1ra Edición. Instituto Nacional de Investigaciones Forestales, Agrícolas y Pecuarias. México. 18p.

Ávila JM, Ascencio G, Maldonado N (2014) Producción y utilización de forraje de soya en bovinos. 1ra Edición. Instituto Nacional de Investigaciones Forestales, Agrícolas y Pecuarias, México. 25p. 
Bautista-Martínez Y, Hernández-Mendo O, Crosby-Galván MM, Joaquin-Cancino S, Ruíz Albarrán M, SalinasChavira J, Granados-Rivera LD (2020) Physicochemical characteristics and fatty acid profile of beef in Northeastern Mexico: grazing vs feedlot systems. CyTA - Journal of Food 18: 147-152.

Belachew Z, Yisehak K, Taye T, Janssens GPJ (2013) Chemical composition and in sacco ruminal degradation of tropical trees rich in condensed tannins. Czech Journal of Animal Science 58: 176-192.

Devi KN, Vyas AK, Singh MS, Singh NG (2011) Effect of bioregulators on growth, yield and chemical constituents of soybean (Glycine max). Journal of Agricultural Science 3: 151-159.

Díaz M, Padilla C, Torres V, González A, Curbelo F, Noda A (2003) Caracterización bromatológica de variedades de soya (Glycine max) en producción de forrajes, forrajes integrales y granos en siembras de verano. Revista Cubana de Ciencia Agrícola 37: 311-317.

Enríquez JF, Meléndez F, Bolaños ED, Esqueda VA (2011) Producción y manejo de forrajes tropicales. 1ra Edición. Instituto Nacional de Investigaciones Forestales, Agrícolas y Pecuarias. México. 405p.

Fehr WR, Caviness CF, Burmood DT, Pennington JS (1971) Stage of development descriptions for soybeans, Glycine max (L.) Merrill. Crop Science 11: 929-931.

Garay JR, Estrada B, Bautista Y, Bernal-Flores A, Mendoza SI, Martínez JC, Sosa E, Joaquín S (2020) Forage yield and quality of buffel ' $\mathrm{H}-17$ ' and Urochloa hybrids at different regrowth ages under semi-arid conditions. Grassland Science 66: 277-284.

Garay-Martínez JR, Joaquín-Cancino S, Estrada-Drouaillet B, Martínez-González JC, Joaquín-Torres BM, LimasMartínez AG, Hernández-Meléndez J (2018) Acumulación de forraje de pasto buffel e híbridos de Urochloa a diferente edad de rebrote. Ecosistemas y Recursos Agropecuarios 5: 573-581.

García E (2004) Modificaciones al sistema de clasificación climática de Köppen. 5ta Edición. Instituto de Geografía- Universidad Nacional Autónoma de México. México. 90p.

Karayilanli E, Ayhan V (2016) Investigation of feed value of alfalfa (Medicago sativa L.) harvestedat different maturity stages. Legume Research-An International Journal 39: 237-247

Liu T, Ren T, White PJ, Cong RH, Lu J (2018) Storage nitrogen co-ordinates leaf expansion and photosynthetic capacity in winter oilseed rape. Journal of Experimental Botany 69: 2995-3007.

Maldonado N (2017) Soya de temporal y riego para el sur de Tamaulipas, ciclo P-V. En: Instituto Nacional de Investigaciones Forestales, Agrícolas y Pecuarias (ed). Agenda Técnica Agrícola de Tamaulipas. Ciudad de México, México. pp: 92-105.

Nkosi BD, Meeske R, Langa T, Motiang MD, Modiba S, Mkhize NR, Groenewald IB (2016) Effects of ensiling forage soybean (Glycine max (L.) Merr.) with or without bacterial inoculants on the fermentation characteristics, aerobic stability and nutrient digestion of the silage by Damara rams. Small Ruminant Research 134: 90-96.

Pérez JA, García E, Enríquez JF, Quero AR, Pérez J, Hernández A (2004) Análisis de crecimiento, área foliar específica y concentración de nitrógeno en hojas de pasto "mulato" (Brachiaria híbrido, cv.). Técnica Pecuaria en México 42: 447-458.

Shamim MJ, Tanaka Y, Sakoda K, Shiraiwa T, Nelson RL (2021) Physiological analysis of leaf photosynthesis of backcross-derived progenies from soybean (Glycine $\max$ (L.) Merrill) and G. tomentella Hayata. Plant Production Science 24: 109-117. 
SMN (2020) Normales climatológicas. Información Climatológica por Estado. Servicio Metrológico Nacional. https://smn.conagua.gob.mx/es/informacion-climatologica-por-estado?estado=slp. Fecha de consulta: 13 de abril de 2021.

Tobía C, Villalobos E (2004) Producción y valor nutricional del forraje de soya en condiciones tropicales adversas. Agronomía Costarricense 28: 17-25.

Tobía C, Villalobos E, Rojas A, Soto H, Moore KJ (2008) Valor nutricional del ensilaje de soja (Glycine max L. Merr.) fermentado con melaza e inoculado con Lactobacillus brevis 3. Livestock Research for Rural Development. http://www.Irrd.org//rrd20/7/tobi20106.htm. Fecha de consulta: 13 de abril de 2021.

Torres-Salado N, García-Raymundo OD, Maldonado-Peralta MA, Rojas-García AR, Ayala-Monter MA, SánchezSantillán P (2020) Productive behavior of sheep fed with soy (Glycine max L.) forage. Agroproductividad 13: 63-67.

Touno E, Kaneko M, Uozumi S, Kawamoto H, Deguchi S (2014) Evaluation of feeding value of forage soybean silage as a substitute for wheat bran in sheep. Animal Science Journal 85: 46-52.

Van Soest, PJ, Robertson JB, Lewis B A (1991) Methods for dietary fiber, neutral detergent fiber, and nonstarch polysacaccharides in relation to animal nutrition. Journal of Dairy Science 74: 3583-3597.

Vargas-Bello-Pérez E, Mustafa AF, Seguin P (2008) Effects of feeding forage soybean silage on milk production, nutrient digestion, and ruminal fermentation of lactating dairy cows. Journal of Dairy Science 91: 229-235. 